\title{
An outbreak of infection with Salmonella typhimurium in a general hospital
}

\author{
BY NAOMI DATTA \\ Department of Bacteriology \\ AND R. B. PRIDIE \\ Department of Medicine, Postgraduate Medical School, Hammersmith Hospital, \\ London, W. 12 \\ With a Note by E. S. ANDERSON \\ Director of the Central Enteric Reference Laboratory and Bureau, Colindale, \\ London, N.W.9
}

(Received 25 February 1960)

Salmonella infections in the population at large are nearly always due to the ingestion of food contaminated with salmonella organisms. The incubation period is short (usually 8-24 hr.) and the great majority of the people affected in an outbreak fall ill within a few hours of one another and usually only a small number of secondary cases occur in the ensuing days or weeks. But in hospitals, salmonella outbreaks with quite a different time sequence sometimes occur.

This paper describes an outbreak of infection in a large general hospital with Salmonella typhimurium, phage-type 27 , in which there was no evidence of foodborne spread and the epidemiological pattern was more like that of hospital crossinfection with staphylococci, streptococci or diphtheria bacilli or even shigellae than that of food poisoning. The importance of this method of spread was recognized in the Public Health Laboratory Service Report of 1953 on food poisoning in England and Wales (Report, 1955) where, in considering some forty salmonella outbreaks in hospitals, it is said: '...cross-infection probably accounted for many'. One such hospital outbreak, in which babies were infected with Salmonella derby has previously been reported by Rubbo (1948). Bate \& James (1958) also reported recurrent outbreaks of $S$. typhimurium infection in a babies' ward which were of peculiar interest epidemiologically. The spread of infection was not food-borne; it was finally traced to infected dust. Cross-infection played only a minor part.

\section{COURSE OF THE OUTBREAK}

The hospital concerned has about 650 beds which are accommodated in two large blocks, North and South (Fig. 1), each with its own kitchen. There are both medical and surgical wards in each of these blocks and an obstetric department in the South and a premature baby unit and children's wards in the North block.

During the first 11 weeks of $1959 \mathrm{~S}$. typhimurium was isolated from three of the hospital patients, a man (who had no symptoms of enteritis), a woman and a baby. 
There was no apparent connexion between these three cases and no article of diet common to them. The cultures from these three patients were not phage-typed and they were not preserved.

On 19 March 1959 S. typhimurium was isolated from two patients, again in separate wards. The phage-type of both strains was type 27. One of the patients was a man of 54 years in Ward D 1, who had been admitted as an emergency for laparotomy 12 days previously and at operation had been found to have an acute pancreatitis. He was in a twenty-four-bedded surgical ward severely ill and developed diarrhoea on 19 March. The other was a married woman aged 18 years in ward D 3, who 4 days previously had given birth to twins. She developed diarrhoea after delivery and two different salmonella sero-types were isolated from her stool specimen of $19 \mathrm{March}-S$. typhimurium and $S$. muenchen. The wards in which these two patients were accommodated were one above the other in the South block; their meals were prepared in the same kitchen but the medical, nursing and domestic staff were quite separate.

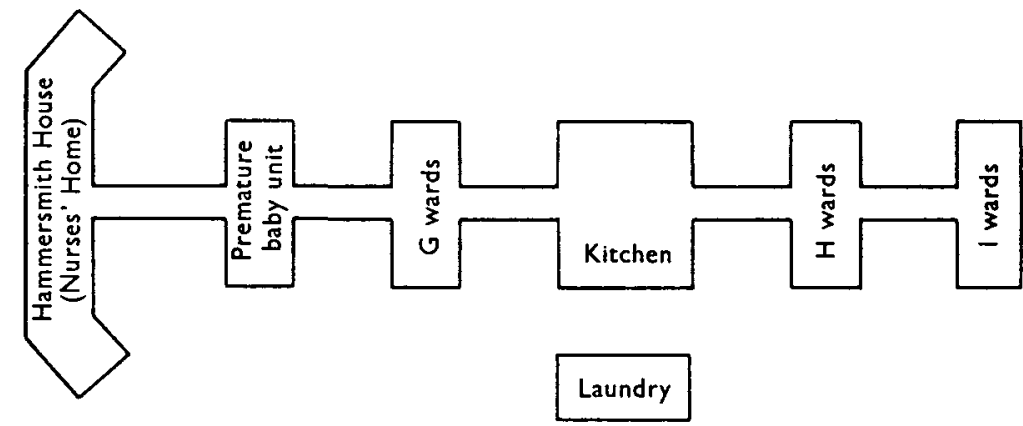

North block

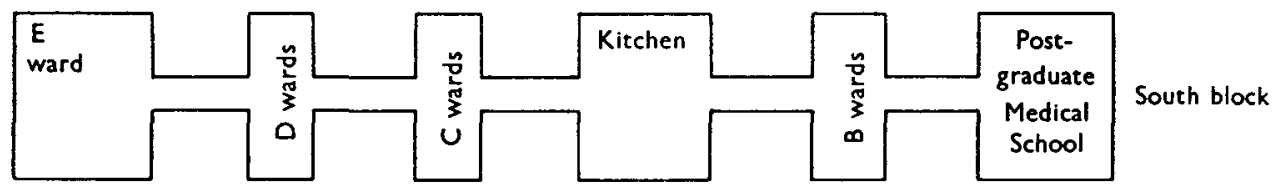

Fig. 1. Simplified plan of hospital showing positions of wards and kitchens.

During the next fortnight twelve further cases of diarrhoea occurred, mostly in wards D 1 and D 3 but they were also scattered widely through both hospital blocks (see Fig. 2). From then on, and for the next 3 months, in spite of strenuous measures to prevent it, the infection spread to nearly every ward of the hospital. During this time 178 patients were shown to be excreting S. typhimurium of the same phage-type, of whom ninety-two had gastro-intestinal symptoms (see Fig. 3). 


\section{Clinical features}

The disease was in most cases mild. The majority of those affected suffered from diarrhoea for less than $24 \mathrm{hr}$., often accompanied by a slight pyrexia. But in twenty-five patients the diarrhoea continued for 2 days or more and a few

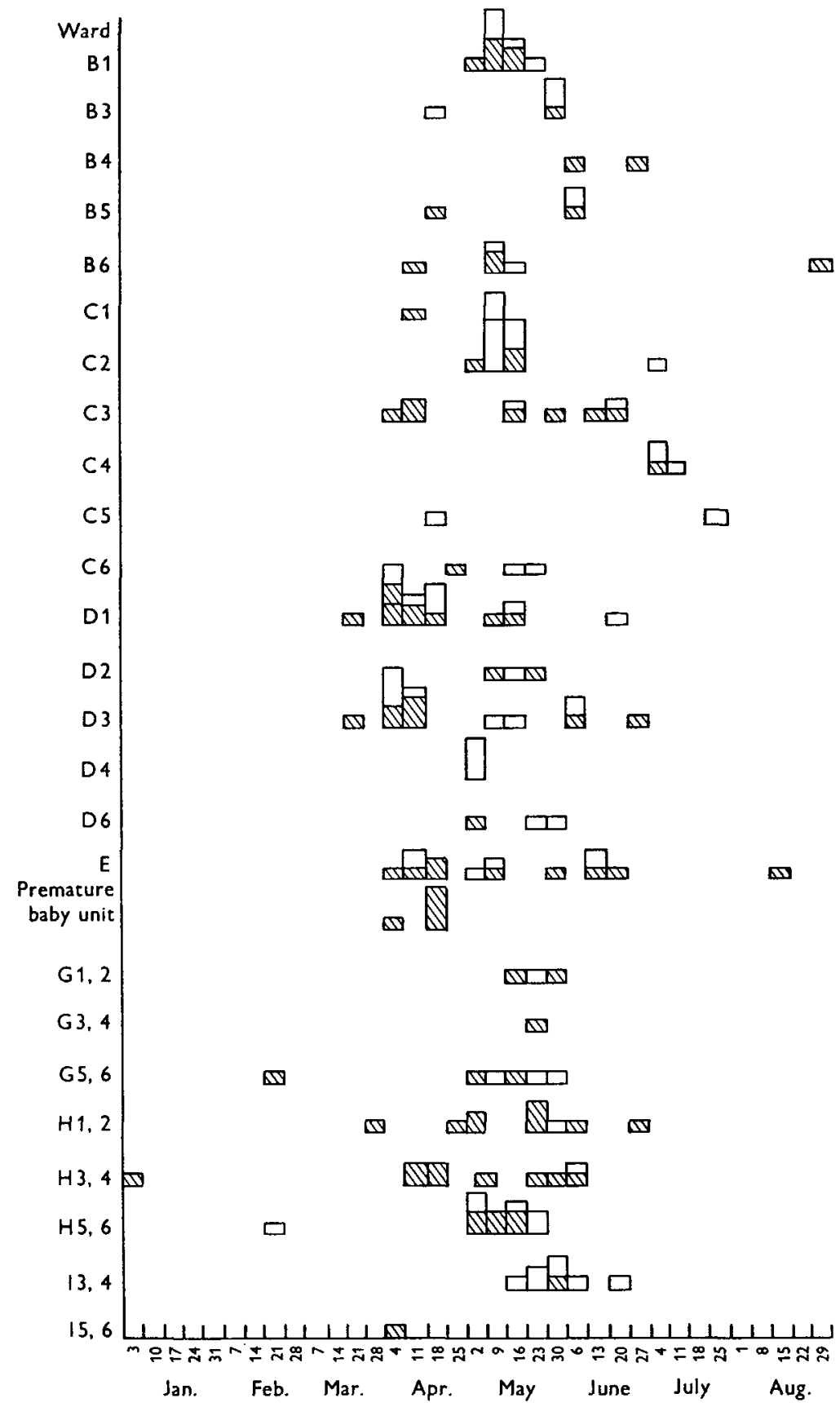

Fig. 2. Showing the distribution of infected patients in the wards.

$\square=$ symptomless excreters; $\square=$ excreters with symptoms. 
complained of severe colic. Vomiting occurred in only fifteen cases and in eight of these it could have been ascribed to a cause other than the salmonella infection. In the maternity and premature baby departments twenty-six new-born babies were shown to be excreting $S$. typhimurium, and of these, thirteen had mild gastrointestinal symptoms; they passed a few loose green stools or vomited once or twice. Only two babies became slightly dehydrated.

Of the 178 excreters ten died, but seven of these deaths were quite unrelated to the salmonella infection. In the other cases the onset of acute diarrhoea in very ill patients appeared to be a contributory cause of death in two, and the immediate cause in the third. This was a man of 54 years with chronic bronchitis and heart failure; he had severe diarrhoea for 2 days followed by paralytic ileus and died $12 \mathrm{hr}$. later.

\section{Symptomless excreters}

A total of 154 symptomless excreters was detected during the outbreak-86 patients, 1 doctor, 57 nurses and 10 domestic staff. It is probable that the numbers were really much higher as experience shows that many carriers are missed unless daily examination of the stools is made, which was, of course, not possible for the whole of the hospital population.

\section{Origin of the infection}

The origin of the infection is unknown. The organism could have been introduced in contaminated food or by an infected person-either a member of the staff or a patient. The culture of the same organism from two patients in separate wards on 19 March suggested that neither of them was the original source. One of the three earlier cases in 1959 might possibly have been; unfortunately the cultures from these patients were not preserved and it is not known if they were of the same strain of $S$. typhimurium.

As part of an extensive search for the source of the infection, the following foodstuffs from the hospital and ward kitchens were examined on many occasions but salmonella organisms were never isolated:

Cooked meats

Cake mix (containing dried egg)

Flour

Babies' dried milk
'Gastric' diets (prepared on ward) 'Complan'

Churn milk

Human milk (refrigerated)

The hospital does not use dried egg other than that included in the cake mix. Of course single batches of any of these might have been infected and have been used up before the investigations began.

From 14 May to the end of July 1959 stool specimens were cultured from every patient on admission to hospital in order to discover whether the infection was endemic in the local population. About 1500 such specimens were received and on two occasions (17 and 23 May) S. typhimurium, phage-type 27, was isolated from patients (with no known previous contact with the hospital) on the day of admission, but as this was fairly late in the outbreak it gave no proof of whether the primary local source was inside or outside the hospital. A suggestion that it may 
have been outside came from the fact that in another institution in the neighbourhood there were cases of infection of $S$. typhimurium, phage-type 27 over the same period.

\section{Examination of the hospital staff}

All members of the nursing, domestic and kitchen staffs, and as many as possible of the medical staff, had stool specimens examined bacteriologically at least once. $S$. typhimurium was never isolated from the kitchen staff (although $S$. potsdam was isolated from one member), even though these examinations were initiated at an early stage in the outbreak and were repeated on many occasions.

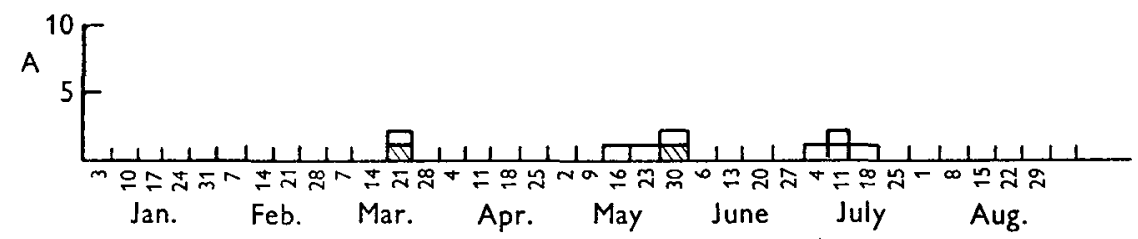

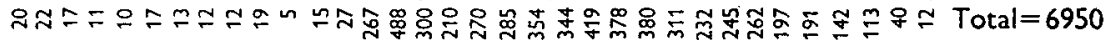

Weekly no. of stool specimens examined ${ }^{\star}$
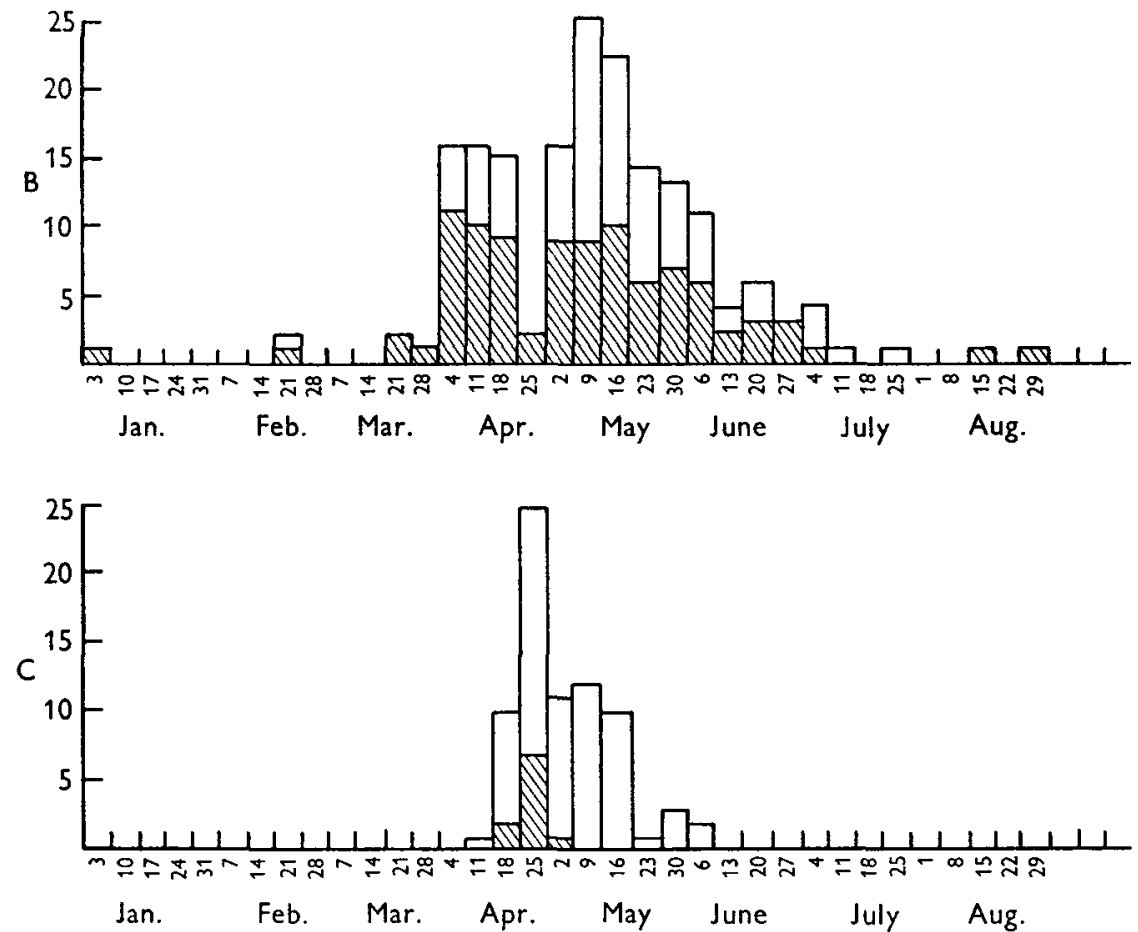

Fig. 3. Histograms showing weekly first isolations of salmonellae over the period January to August 1959. A, refers to first isolations from patients of salmonellae other than $S$. typhimurium, phage-type 27 . B, refers only to first isolations from patients of S. typhimurium, phage-type 27. C, refers to first isolations from the staff of $S . t y p h i$ murium, phage-type 27. $\square=$ symptomless excreters. $\triangle \mathbb{W}=$ excreters with symptoms.

* These figures refer to specimens examined at the Postgraduate Medical School. 
The organism was isolated from only one member of the medical staff and from none of the senior nurses. On the other hand, sixty-five of the junior nurses were found at some time to be excreting $S$. typhimurium, and two excreted S. muenchen; of these sixty-seven infected nurses, ten suffered from short attacks of diarrhoea, the remainder were symptomless. Ten of the domestic staff (including four laundry workers) were found to be excreting $S$. typhimurium, and one ward orderly, $S$. muenchen. It will be seen from Fig. 3 that $S$. typhimurium was isolated from no member of the staff until the week end, 11 April, although many specimens from contacts among them had been examined during the previous 2 weeks.

\section{METHODS OF CONTROL}

Every effort was made to improve the standards of personal and kitchen hygiene throughout the hospital and the importance of hand-washing was impressed on all, patients and staff alike. Bowls of disinfectant were provided at all wash-hand basins and everyone was asked to immerse his or her hands in the disinfectant after washing, but before drying them.

No made-up food was kept on the wards and patients were denied private food supplies other than fruit. The possibility of contamination of food by rodents was considered but searches failed to reveal any rats or mice.

In attempting to control the outbreak and to stop the spread of the infection the main principle was to move every infected person out of the wards. Patients were sent away, either to their homes or to isolation hospitals and staff were put off work. Where, for some reason, patients could not be moved they were isolated and barrier-nursed, but isolation facilities were very limited. Faecal specimens were obtained from all ward contacts and examined bacteriologically. Contacts among the patients were given phthalyl sulphathiazole as a prophylactic, but contacts among the staff were not treated. Infected persons, whether patients or staff, were usually treated with a combination of phthalyl sulphathiazole and streptomycin by mouth but combinations of other suitable drugs were sometimes used.

Any effect of such treatment on the duration of carriage of the organism by the patients could not be assessed since follow-up was not practicable in most cases. With regard to the nurses an attempt was made; at the height of the outbreak it was decided to test the effect of the sulphonamide-streptomycin treatment on the length of salmonella carriage by giving the odd numbers, of the nurses found to be excreters, the drug treatment and the even numbers inactive tablets. But before significant numbers could be collected the outbreak came to an end. However, fifty-seven nurses who were excreting the organism were treated with phthalyl sulphathiazole $2 \mathrm{~g}$. 6 -hourly and streptomycin $2 \mathrm{~g}$. daily by mouth and this continued for 5 days. With this treatment forty-four nurses were negative on repeated culture from 14 days onwards but thirteen were still excreting the organism.

This proportion is lower than that reported by Kwantes (1952) for the proportion of excreters who were positive 2 weeks after an explosive outbreak of $S$. typhimurium food poisoning, but the difference cannot be attributed with certainty to the treatment. 
No nurse was found to excrete the organism for longer than 10 weeks.

After the end of May 1959 the outbreak gradually died down. By comparing the number of new isolations with the total number of specimens cultured (Fig. 3) it can be seen that the disappearance of the organism was not a reflexion of a reduction in the number of faecal specimens examined. Since the end of August $1959 \mathrm{~S}$. typhimurium has not been isolated from specimens originating from any patient or member of the staff of the hospital. Between September and the end of the year (1959) well over 300 faecal specimens from the medical, nursing and domestic staff were cultured at the Postgraduate Medical School to determine whether the carriage of the organism still continued. S. typhimurium was not isolated from any of these specimens.

\section{BACTERIOLOGICAL INVESTIGATIONS \\ Culture of faeces}

Faeces from the hospital patients were examined in the Department of Bacteriology of the Postgraduate Medical School but during the outbreak those from the hospital staff were examined at the Public Health Laboratory, County Hall, S.E.1.

At the Postgraduate Medical School faeces specimens are normally plated directly on MacConkey and desoxycholate citrate agar media and also inoculated into Selenite F broth, incubated overnight and subcultured on to MacConkey plates the next day. This method was continued until 20 May, by which time the volume of work was getting too much for the resources of the department. At the same time it was clear that the majority of the salmonella isolations were made from the enrichment medium and not from direct plating procedures. It was therefore decided to discontinue direct plating for the duration of the outbreak, in spite of the fact that the change meant $24 \mathrm{hr}$. delay in reporting some of the positive cultures.

The Public Health Laboratory at County Hall, in examining specimens from the hospital staff, employed the Selenite enrichment method only.

Of 223 salmonella isolations from hospital patients over the period 19 March to 20 May 1959, 91 were made from direct platings and 132 were obtained only after enrichment through Selenite broth. But these figures include the results of many follow-up specimens; the comparable figures for first isolations are given in Table 1.

Table 1. Record of first isolations of Salmonella typhimurium from patients 19 March to 20 May 1959

$\begin{array}{ccc} & \begin{array}{c}\text { On } \\ \text { direct plating }\end{array} & \begin{array}{c}\text { From } \\ \text { Selenite only }\end{array} \\ \text { Patients with symptoms } & 41 & 25 \\ \text { Patients without symptoms } & 14 & 38 \\ \text { Total } & 55 & 63\end{array}$

During the course of the outbreak 6950 stool specimens from patients were cultured and apart from $S$. typhimurium, phage-type 27, other salmonellae were isolated from ten of the patients, only two of whom had symptoms of diarrhoea. 
The salmonella sero-types were:

$\begin{array}{ll}\text { S. muenchen } & 4 \text { isolations } \\ \text { S. thompson } & 3 \text { isolations } \\ \text { S. typhimurium (untypable) } & 1 \text { isolation } \\ \text { S. typhimurium (type U 40) } & 1 \text { isolation } \\ \text { S. anatum } & 1 \text { isolation }\end{array}$

\section{Culture of other possible sources of infection}

Because of the time distribution of the infections and because of the negative findings from the bacteriological examinations of the kitchen staff and the various foodstuffs from the hospital kitchens, it seemed likely that cross-infection was occurring either directly from person to person or via dust or fomites or both. In ward D 1, where one of the first cases occurred, every man who was well enough to get up to go to the toilet, became infected. Cultures of swabs from the lavatory door handles, chain handles and seats, however, failed to yield growths of $S$. typhimurium. Exposed plates on the floor round the lavatory pan were also negative (see Darlow \& Bale, 1959). Inquiries were made about any food being shared between these ambulant men (who were in close social contact) which might have provided a culture medium for the organism, but they had had none, other than that supplied by the hospital kitchen which was eaten at once.

Ward dust taken from the vacuum cleaner of ward D 1 was cultured in Selenite broth and yielded S. typhimurium, phage-type 27. Dust from the vacuum cleaners in all the other wards was repeatedly cultured but the organism was never again isolated from this source.

Aerial contamination was indicated at a later date during the examination of a chest ward where two patients were suffering from diarrhoea. Sputum from fourteen patients in this ward was cultured in Selenite broth and S. typhimurium was isolated from five of these specimens. None of the patients whose sputum was positive was, at the time, excreting the organism in his faeces.

The isolation of the organism from the dust of the ward and from the sputum of patients clearly indicate that aerial spread of the infection can occur. Air sampling through slit-samplers was carried out at various times, the air being collected from a number of different places in the wards, including beside the beds of infected patients while their beds were being made, but none of the samples yielded salmonellae.

Contaminated sheets. Indications that contaminated bed sheets could spread infection came from the fact that several laundry workers became infected, and that, among the domestic staff in the nurses home the only ones who contracted the infection were those who collected the used sheets to send to the laundry.

Culture of sheets, bedspreads and cubicle curtains by pulling an opened Petri dish, containing a selective medium, across the taut surface of the material (Blowers \& Wallace, 1955) and incubating the plates overnight failed to yield any growth of S. typhimurium.

The organism was, however, isolated from sheets used by symptomless excreters 
by immersing the whole sheet in buffered water, then filtering the water through a layer of filter-aid (Hyflo Supercel) on a Berkefeld filter and culturing the whole of the filter cake in Selenite broth.

Control sheets used by uninfected patients, treated in the same way failed to yield any growth of salmonella organisms. Culture of towels in common use by ward staff also gave negative results.

Experimental work on the survival in ward dust and on sheets of this particular strain of S. typhimurium, and of $S$. muenchen isolated at the same time, is in progress.

\section{DISCUSSION}

It is bulieved that once $S$. typhimurium had gained access to the hospital commutiny, by some method unknown, it then spread by human contact and through dust and aerial contamination. The final mode of entry must have been the swallowing of the organism which may, in many cases, have been from taking food or drink which had been handled on the wards where the organism was widely distributed. Such immediate contamination of food, without opportunity for multiplication of microbes, is considered here as an example of cross-infection; the infection it may cause is quite different epidemiologically from food-borne infection in the usual sense, where an article of food acts as a culture medium for a pathogenic organism and its consumption results in an explosive outbreak of enteritis.

The evidence against large scale food-borne spread was:

The outbreak was not explosive, but was drawn out over many weeks.

The outbreak involved people such as new-born babies and patients after gastric surgery who shared no article of diet with the community.

No member of the kitchen staff was ever shown to be excreting the organism despite repeated culturing of their stools. In a food-borne outbreak it would be surprising if no one working in the kitchen became infected.

The cases were evenly distributed in both hospital blocks, although each block had its own separate kitchen.

No item of food was traced which seemed to be a likely source of infection and repeated culture of the various foods used, failed to yield any salmonella organisms.

Although this negative evidence could not, by itself, be accepted as proof that the outbreak was not food-borne there is significant evidence to support the view that direct contact and aerial spread were responsible for the epidemiological pattern. The important points of which are:

The time sequence of events.

The isolation of the organism from ward dust, sheets of infected patients, sputum of patients who were not themselves excreting the organism from the bowel.

The infection of laundry and domestic workers, who handled sheets from infected persons. 
The lack of infection among the doctors and senior nurses, who have less contact with the patients than do the junior nurses, many of whom became infected.

The experimental studies of McCullough \& Eisele $(1951 a, b)$ on human infections with various salmonella strains showed that, although the ingestion of fairly large doses (usually more than $10^{6}$ bacilli) was required to produce a clinical illness in healthy adults, even the smallest doses tested (about $10^{3}$ bacilli) could give rise to a transient carrier state. If the spread of infection in the outbreak under consideration was in fact due to what is generally known as cross-infection, the infecting dose might be much smaller than the dose found by McCullough \& Eisele to be required to initiate clinical symptoms. This is, however, pure speculation and although it is reasonable to assume that the infecting dose for patients, whose primary illness might be quite serious, would be much less than for healthy people, reliable knowledge on this point is not available. Indeed there are so many gaps in our understanding of the infectivity of salmonellae that it is not possible to give any reliable figure for the infecting dose in man. According to McCullough \& Eisele, different strains of the same organism, given by mouth under the same experimental conditions, may show a 50-fold difference in their infecting dose. It would be reasonable to believe that there may exist strains of salmonellae able to produce symptoms when swallowed in quite small numbers. McCullough \& Eisele did not test S. typhimurium, which is the species most frequently found in human infections, certain strains of which may have a small infecting dose. It is of interest to recall that $S$. muenchen was introduced into the hospital at about the same time as $S$. typhimurium, phage-type 27 , but did not spread to anything like the same extent.

Reports on the isolation of salmonellae from the tonsils of children (Hormaeche, Peluffo \& Aleppo, 1940; Varela \& Olarte, 1942) are of interest to the present investigation although the significance of these findings is by no means clear.

In 1952 Dr Joan M. Boissard and Dr R. M. Fry of the Public Health Laboratory, Cambridge (personal communication) investigated a hospital outbreak of S. typhimurium infection with a very similar epidemiological pattern to the one described here. A patient was admitted to the hospital in February 1952 and was later found to be infected with S. typhimurium. From the middle of March until July, 165 patients, 143 nurses and 58 of the domestic staff were found to be infected.

From a detailed study of the method of spread Boissard \& Fry concluded that food had not been contaminated in bulk in the kitchen, but that infection was being spread directly from patient to patient, between patients and staff, and between members of the staff themselves. The main method of spread appeared to be the comparatively light faecal contamination of such articles as biscuits and bread and butter, handled by persons with unwashed, or insufficiently washed, hands. Methods used to control the outbreak were eventually successful.

It has been found by others that the patient excreting salmonellae infects his environment; for example, Dr A. J. H. Tomlinson (personal communication) isolated $S$. paratyphi $B$ from ward dust and from the bedding of patients infected with this organism, using the 'vacuum cleaner' technique of Williams (1949) (see 
also, Bate \& James (1958)). Such infection of the patient's environment is probably more important than is often recognized, particularly in institutions, where many people are exposed to the same environment. In the outbreak described here it must be emphasized that a high proportion of those infected had an unrelated primary illness, and they may therefore have been more susceptible to salmonella infections than healthy subjects. While this may explain the cross-infection in the patients, it does not explain the clinical infection of a number of the nurses looking after those patients.

Once it was realized that $S$. typhimurium was spreading amongst the hospital population, vigourous measures were instituted to control the spread, and ultimately succeeded in bringing the epidemic to a close. Unfortunately, it was not recognized, when S. typhimurium was isolated from the first three apparently unconnected persons, that these cases might represent the beginning of a hospital outbreak, and by the time control measures were instituted the organism was widely distributed. Had the first three strains been phage-typed, we should have been in a much better position to appreciate their significance.

\section{SUMMARY}

An outbreak of Salmonella typhimurium infection in a large general hospital is described. The outbreak was spread over some 20 weeks and gave rise to 102 cases of enteritis and there were at least 150 symptomless excreters of the organism.

Evidence is presented to support the view that the method of spread was by cross-infection, not by contamination of food in bulk. Control measures, which ultimately brought the outbreak to an end, were instituted on this basis.

We wish to thank Dr A. J. H. Tomlinson and his staff at the Public Health Laboratory, County Hall, S.E. 1 for the generous assistance they gave us in examining all the specimens from the hospital staff during the outbreak. The resources of the Department of Bacteriology of the Postgraduate Medical School were at that time largely devoted to teaching and adequate investigations could not have been made without this help. Dr Tomlinson also gave us much advice both during and after the outbreak for which we are most grateful.

Dr E. S. Anderson, Director of the Central Enteric Reference Laboratory and Bureau, Colindale, London, N.W. 9, very kindly undertook the phage-typing of many of the strains of Salmonella typhimurium isolated and we wish to express our thanks to him for this and other help he gave us in the investigation of the outbreak.

Our thanks are also due to Dr Joan Taylor, Director of the Salmonella Reference Laboratory, Colindale, for her help and interest.

Discussions with Dr R. M. Fry and Dr Joan Boissard in Cambridge in connexion with their investigations of a similar outbreak were most interesting and valuable and it gives us pleasure to thank them.

We also acknowledge gratefully the help and co-operation of our colleagues at Hammersmith Hospital, especially Dr C. E. Roberts, the Medical Superintendent, and of those at the Postgraduate Medical School. 


\section{REFERENCES}

Bate, J. G. \& J JMes, Ursula (1958). Lancet, ii, 713.

Blowers, A. R. \& Wallace, K. R. (1955). Lancet, i, 1250.

Darlow, H. M. \& Bate, W. R. (1959). Lancet, i, 1196.

Hormaeche, E., Peluffo, C. A. \& Alepro, P. L. (1940). Arch. Paediat. Uruguay, $11,8$.

Kwantes, W. (1952). Mon. Bull. Minist. Hlth Lab. Ser. 11, 239.

McCullough, N. B. \& Eisele, C. W. (1951a). J. infect. Dis. 88, 278.

McCullough, N. B. \& Eisele, C. W. (1951b). J. infect. Dis. 89, 209.

Report (1955). Mon. Bull. Minist. Htth Lab. Ser. 14, 34.

RuBbo, S. D. (1948). J. Hyg., Camb., 46, 158.

VARELA, G. \& Olarte, J. (1942). Rev. Inst. Salubp. Enferm. trop., Méx., 3, 289.

Williams, R. E. O. (1949). J. Hyg., Camb., 47, 416.

\section{NOTE BY E. S. ANDERSON}

The incidence of Salmonella typhimurium, phage-type 27

Salmonella typhimurium, phage-type 27, the organism responsible for the outbreak described in the foregoing paper, is of special interest for a number of reasons. It is only during the last 3 years that we have been able to recognize this type. It has caused at least seven epidemics, of which that at Hammersmith Hospital was probably the largest. Very few sporadic infections have been found. Indeed, although some hundreds of cases are known to have occurred in epidemics, only ten appeared to be sporadic. Most of the outbreaks were in hospitals, and although no connexion can be postulated between some of these incidents, work carried out in this laboratory suggests that distant spread through human agency may have carried the infection to outlying areas. Such spread may have occurred not only during the period of the Hammersmith outbreak, but also, through the agency of symptomless excreters, before the infection was clinically manifest in Hammersmith Hospital.

The fact that the infection persisted for long periods in affected hospitals suggests that, apart from the probability that symptomless excretion was common, the environmental contamination demonstrated at Hammersmith Hospital was also present in the other institutions concerned. It seems probable, moreover, that this organism has a high communicability, that the dose required to produce infection is small, as suggested by the authors of the foregoing paper, and that the epidemiology of these infections resembles that of dysentery rather than salmonellosis, at any rate as far as all but the first cases in hospital outbreaks are concerned.

In spite of the considerable amount of human infection caused by type 27 , it has never been isolated either from animals or from foodstuffs. Furthermore, apart from those epidemics known or suspected to be connected with each other, there is, up to the present, no hint concerning the materials that acted as the source and vehicles of the original organisms which instituted the foci forming the first links of the chain of infection. The large-scale importation of foods from foreign countries has posed a number of new problems in salmonellosis. In one instance, imported meat yielded a phage-type of $S$. typhimurium that had caused considerable human outbreaks but had never been found in home-grown food. It is possible that phage-type 27 enters this country in a similar fashion. 
I should like to point out here that it is only during the last 2 or 3 years that the revised phage-typing scheme for $S$. typhimurium, which owes so much of its development to the late Miss Bessie Callow (Callow, 1959), ${ }^{*}$ has made possible the identification of such types as the one in question. Before the extended range of phages was available, 27 was one of a considerable number of types that were grouped together as being untypable.

* Callow, Bessie R. (1959). J. Hyg., Camb., 57, 346. 